\title{
RHODIUM-CATALYZED ENANTIOSELECTIVE DIBORATION OF SIMPLE ALKENES.
}

\author{
Jeremy B. Morgan, Steven P. Miller and James P. Morken" \\ Department of Chemistry, Venable and Kenan Laboratories \\ University of North Carolina, Chapel Hill, NC 27599-3290
}

\section{Supplementary Material}

General. ${ }^{1} \mathrm{H}$ NMR spectra were recorded on Bruker DRX $(400 \mathrm{MHz}$ or 300 $\mathrm{MHz}$ ). Chemical shifts are reported in ppm from tetramethylsilane with the solvent resonance as the internal standard $\left(\mathrm{CDCl}_{3}: 7.24 \mathrm{ppm}\right)$. Data are reported as follows: chemical shift, integration, multiplicity $(\mathrm{s}=$ singlet, $\mathrm{d}=$ doublet, $\mathrm{t}=$ triplet, $\mathrm{q}=$ quartet, $\mathrm{br}$ = broad, $\mathrm{m}=$ multiplet), coupling constants $(\mathrm{Hz})$ and assignment. ${ }^{13} \mathrm{C}$ NMR were recorded on a Bruker DRX $400(100 \mathrm{MHz})$ spectrometer with complete proton decoupling. Chemical shifts are reported in ppm from tetramethylsilane with the solvent as the internal standard $\left(\mathrm{CDCl}_{3}: 77.0 \mathrm{ppm}\right)$. High resolution mass spectrometry was performed by the University of Minnesota Mass Spectrometry Service Laboratory. Infrared (IR) spectra were obtained using a ASI ReactIR 1000.

Liquid chromatography was performed using forced flow (flash chromatography) on silica gel $\left(\mathrm{SiO}_{2}, 32\right.$ to $\left.63 \mu \mathrm{m}\right)$ purchased from Scientific Adsorbents, Inc. Thin layer chromatography (TLC) was performed on EM science $0.25 \mathrm{~mm}$ silica gel 60 plates. Visualization was achieved UV light or phosphomolybdic acid in ethanol followed by heating.

Analytical gas-liquid chromatography (GLC) was performed on a Hewlett-Packard 6890 Series chromatograph equipped with a CTC Analysis Combi Pal autosampler by Leap Technologies (Carrboro, NC), a split mode capillary injection system, a flame ionization detector and a Supelco $\beta$-dex 120 column with helium as the carrier gas. Analytical high performance liquid chromatography (HPLC) was performed on a Shimadzu liquid chromatograph equipped with a UV detector and either a Chiralcel OJ or a Chiralcel OD column.

All reactions were conducted in oven and flame dried glassware under an inert atmosphere of argon. Alkene starting materials were all commercially available. [(nbd)Rh(acac)], bis(catecholato)diboron, and THF were purchased from Aldrich Chemical Company and (S)-Quinap was purchased from Strem Chemical Company. All reagents were used as received. 


\section{Representative Procedure for Catalytic, Enantioselective Diboration Reaction}

An oven-dried $20 \mathrm{~mL}$ vial equipped with a stir-bar was charged with $7.4 \mathrm{mg}$ (0.025 mmol) of (bicyclo[2.2.1]hepta-2,5-diene)-(2,4-pentanedionato)-rhodium(I), 11.1 $\mathrm{mg}(0.025 \mathrm{mmol})$ of $(S)$-Quinap, and $1.0 \mathrm{~mL}$ of THF under an inert atmosphere of argon in a dry-box. The resultant yellow solution was stirred for 5 minutes. After this time, $132 \mathrm{mg}(0.55 \mathrm{mmol})$ of bis(catecholato)diboron was added to the solution under argon. The solution turned immediately from yellow to dark brownish-red. The solution was allowed to stir for 5 minutes. After this time, $71 \mathrm{mg}(0.50 \mathrm{mmol})$ of trans-5-decene was added to the solution under argon. The vial was sealed with a screw-cap and removed from the dry box, where the solution was allowed to stir for 15 hours at ambient temperature. After this time, $1 \mathrm{~mL}$ of THF was added to the solution, followed by dropwise addition of $0.800 \mathrm{~mL}$ of $3 \mathrm{M} \mathrm{NaOH}$ and then $0.800 \mathrm{~mL}$ of $30 \% \mathrm{H}_{2} \mathrm{O}_{2}$ dropwise (for hazards associated with $\mathrm{H}_{2} \mathrm{O}_{2}$ see, Encyclopedia of Reagents for Organic synthesis, L.A. Paquette, Ed. John Wiley and Sons, New York, 1995, Volume 4, 2731). The solution was allowed to stir at ambient temperature for 3 hours. The solution was then quenched with $1 \mathrm{~mL}$ of saturated aqueous $\mathrm{Na}_{2} \mathrm{~S}_{2} \mathrm{O}_{3}$ and $10 \mathrm{~mL}$ of $1 \mathrm{M} \mathrm{NaOH}$. The mixture was extracted with ethyl acetate $(3 \times 25 \mathrm{~mL})$ and the combined organic layers were washed with brine $(1 \times 10 \mathrm{~mL})$. The organic layers were then dried over anhydrous $\mathrm{MgSO}_{4}$, filtered, and the solvent removed by rotary evaporation. The crude material was purified by silica gel chromatography $(2: 1$ hexanes : ethyl acetate) to provide $71 \mathrm{mg}$ $(81 \%)$ of pure $(5 R, 6 R)$-decane-5, 6-diol.

\section{Representative Procedure for Large-Scale Catalytic, Enantioselective Diboration Reaction}

An oven-dried $50 \mathrm{~mL}$ round-bottom flask equipped with a stir-bar was charged with $11.9 \mathrm{mg}$ (0.041 mmol) of (bicyclo[2.2.1]hepta-2,5-diene)-(2,4-pentanedionato)rhodium(I), $17.8 \mathrm{mg}(0.041 \mathrm{mmol})$ of $(S)$-Quinap, and $2.0 \mathrm{~mL}$ of THF under an inert atmosphere of argon in a dry-box. The resultant yellow solution was stirred for 5 minutes. After this time, $2.12 \mathrm{~g}(8.90 \mathrm{mmol})$ of bis(catecholato)diboron was added to the solution under argon. The solution turned immediately from yellow to dark brownishred. The suspension was allowed to stir for 5 minutes. After this time, $1.14 \mathrm{~g}(8.09$ mmol) of trans-5-decene was added to the solution under argon. The vial was sealed with a screw-cap and removed from the dry box, where the solution was allowed to stir for 4 hours at ambient temperature. At this time, an additional $2 \mathrm{~mL}$ of THF was added and the solution was allowed to stir at ambient temperature for 12 hours. After this time, $16 \mathrm{~mL}$ of THF was added to the solution, followed by $27 \mathrm{~mL}$ of $3 \mathrm{M} \mathrm{NaOH}$ dropwise and then $27 \mathrm{~mL}$ of $30 \% \mathrm{H}_{2} \mathrm{O}_{2}$ dropwise with caution. The solution was allowed to stir at ambient temperature for 3 hours. The solution was then quenched with $15 \mathrm{~mL}$ of saturated aqueous $\mathrm{Na}_{2} \mathrm{~S}_{2} \mathrm{O}_{3}$ and $50 \mathrm{~mL}$ of $1 \mathrm{M} \mathrm{NaOH}$. The mixture was extracted with ethyl acetate $(3 \times 150 \mathrm{~mL})$ and the combined organic layers were washed with $1 \mathrm{M}$ $\mathrm{NaOH}(1 \times 50 \mathrm{~mL})$, water $(1 \times 50 \mathrm{~mL})$ and brine $(1 \times 50 \mathrm{~mL})$. The organic layers were then dried over anhydrous $\mathrm{MgSO}_{4}$, filtered, and the solvent removed by rotary evaporation. The crude material was purified by silica gel chromatography $(2: 1$ hexanes : ethyl acetate) to provide $968 \mathrm{mg}(69 \%)$ of pure $(5 R, 6 R)$-decane-5, 6-diol. 


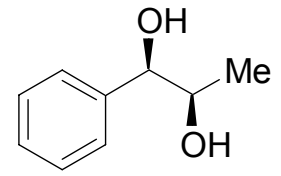

(1R, 2R)-1-phenyl-propane-1,2-diol. IR (neat): 3384 (br, s), 1455 (s), $1038(\mathrm{~s}) \mathrm{cm}^{-1} ;{ }^{1} \mathrm{H}$ NMR: $\delta$ 7.25-7.42 $(5 \mathrm{H}, \mathrm{m}$, aromatic), $4.36(1 \mathrm{H}$, $\mathrm{d}, \mathrm{J}=7.6 \mathrm{~Hz}, \mathrm{ArCHOH}), 3.85(1 \mathrm{H}, \mathrm{qd}, \mathrm{J}=7.2 \mathrm{~Hz}, 6.4 \mathrm{~Hz}$, $\left.\mathrm{CH}_{3} \mathrm{CHOH}\right), 2.44\left(2 \mathrm{H}\right.$, broad s, OH), $1.05\left(3 \mathrm{H}, \mathrm{d}, \mathrm{J}=6.4 \mathrm{~Hz}, \mathrm{CH}_{3}\right)$; ${ }^{13} \mathrm{C}$ NMR: $\delta$ 141.0, 128.5, 128.2, 126.8, 79.5, 72.2, 18.8. HRMS (FAB) Calc'd for $\mathrm{C}_{9} \mathrm{H}_{12} \mathrm{O}_{2}\left(\mathrm{M}+\mathrm{NH}_{4}\right)^{+}: 170.1181$ Found $\left(\mathrm{M}+\mathrm{NH}_{4}\right)^{+}: 170.1177$.

Proof of Stereochemistry. Stereochemical ratios were determined in comparison to authentic racemic materials prepared by osmium tetraoxide dihydroxylation. Relative stereochemistry determined in comparison to ${ }^{1} \mathrm{H}$ NMR reported for the syn diol (Norrby, P-O.; Becker, H.; Sharpless, K. B. J. Am. Chem. Soc. 1996, 118, 35). Absolute stereochemistry established in comparison to authentic $1 \mathrm{R}, 2 \mathrm{R}$ isomer prepared via a Sharpless asymmetric dihydroxylation (Norrby, P-O.; Becker, H.; Sharpless, K. B. J. Am. Chem. Soc. 1996, 118, 35).

Chiral GLC ( $\beta$-dex, Supelco, $\left.140^{\circ} \mathrm{C}\right)$ analysis of diol product:

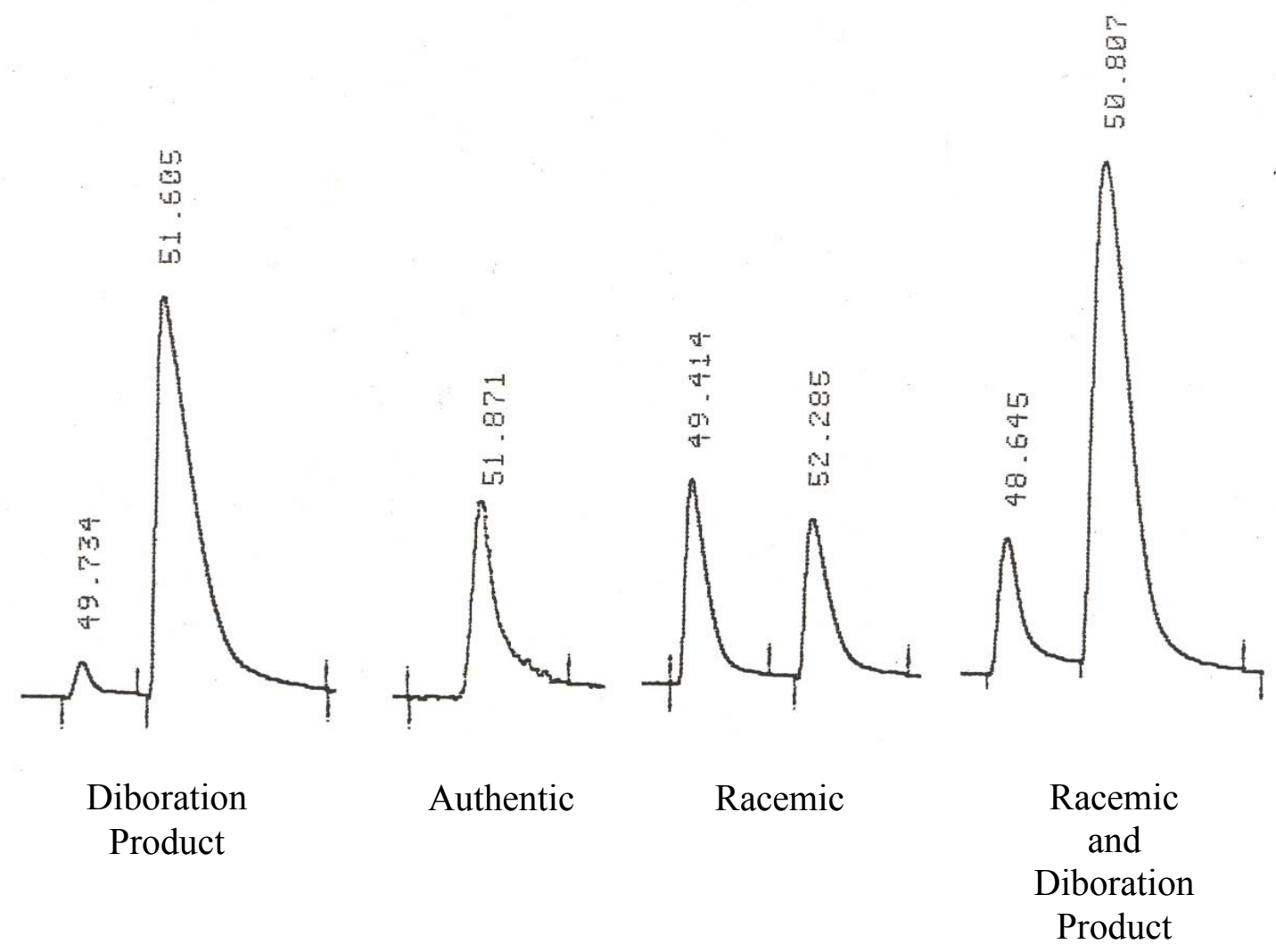


$\mathrm{C}_{4} \mathrm{H}_{9}^{\mathrm{OH}} \mathrm{O}_{\mathrm{OH}}^{\mathrm{H}_{9}}$

(5R, 6R)-decane-5, 6-diol. IR (neat): 3477 (br, s), 1640 (s) $\mathrm{cm}^{-1} ;{ }^{1} \mathrm{H}$ NMR: $\delta 3.39\left(2 \mathrm{H}\right.$, br s, $\left.\mathrm{CH}_{2} \mathrm{CHOH}\right), 1.92(2 \mathrm{H}$, br s, OH $), 1.54-1.25$ $\left(12 \mathrm{H}, \mathrm{m},-\mathrm{CH}_{2^{-}}\right), 0.89\left(6 \mathrm{H}, \mathrm{t}, \mathrm{J}=7.0 \mathrm{~Hz}, \mathrm{CH}_{3}\right) ;{ }^{13} \mathrm{C}$ NMR: $\delta 74.5$, 33.3, 27.8, 22.7, 14.0. HRMS (FAB) Calc'd for $\mathrm{C}_{10} \mathrm{H}_{22} \mathrm{O}_{2}\left(\mathrm{M}+\mathrm{NH}_{4}\right)^{+}$: 192.1964 Found $\left(\mathrm{M}+\mathrm{NH}_{4}\right)^{+}: 192.1958$.

Proof of Stereochemistry. Stereochemical ratios were determined in comparison to authentic racemic materials prepared by osmium tetraoxide dihydroxylation. Relative stereochemistry determined in comparison to ${ }^{1} \mathrm{H}$ NMR reported for the syn diol (Choudary, B. M.; Chowdari, N. S.; Jyothi, K.; Kantam, M. L. J. Am. Chem. Soc. 2002, 124, 5341). Absolute stereochemistry established in comparison to authentic 1R, 2R isomer prepared via a Sharpless asymmetric dihydroxylation (Sharpless, K. B.; Amberg, W.; Bennani, Y. L.; Crispino, G. A.; Hartung, J.; Jeong, K.-S.; Kwong, H.-L.; Morikawa, K.; Wang, Z.-M.; Xu, D.; Zhang, X.-L. J. Org. 1992, 57, 2768).

Chiral GLC ( $\beta$-dex, Supelco, $\left.110^{\circ} \mathrm{C}\right)$ analysis of diol product:

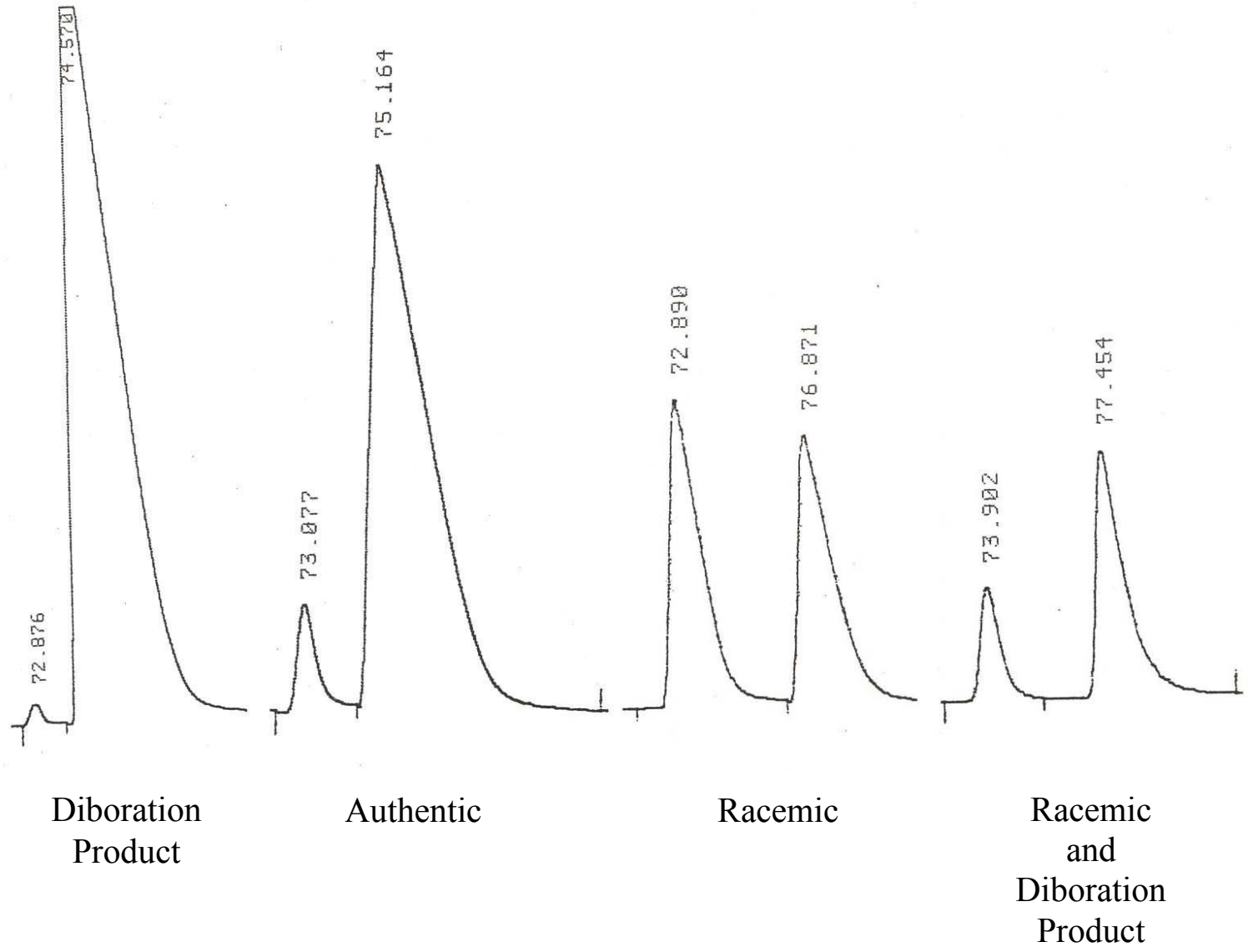


<smiles>OC(c1ccccc1)C(O)c1ccccc1</smiles>

(1R, 2R)-1, 2-diphenyl-ethane-1, 2-diol. IR (neat): 3475 (br, s), $1640(\mathrm{~s}) \mathrm{cm}^{-1} ;{ }^{1} \mathrm{H}$ NMR: $\delta$ 7.24-7.12 $(6 \mathrm{H}, \mathrm{m}, \mathrm{Ph}), 7.11-7.01(4 \mathrm{H}, \mathrm{m}$ $\mathrm{Ph}), 4.67(2 \mathrm{H}, \mathrm{s}, \mathrm{PhCHOH}), 2.95(2 \mathrm{H}, \mathrm{br} \mathrm{s}, \mathrm{OH}) ;{ }^{13} \mathrm{C} \mathrm{NMR}: \delta$ 139.8, 128.1, 127.9, 126.9, 79.1. HRMS (FAB) Calc'd for $\mathrm{C}_{14} \mathrm{H}_{14} \mathrm{O}_{2}\left(\mathrm{M}+\mathrm{NH}_{4}\right)^{+}: 232.1338$ Found $\left(\mathrm{M}+\mathrm{NH}_{4}\right)^{+}: 232.1337$.

Proof of Stereochemistry. Stereochemical ratios were determined in comparison to authentic racemic materials prepared by osmium tetraoxide dihydroxylation. Relative stereochemistry determined in comparison to ${ }^{1} \mathrm{H}$ NMR reported for the syn diol (Wang, Z.-M.; Sharpless, K. B. J. Org. Chem. 1994, 59, 8302). Absolute stereochemistry established in comparison to commercially available authentic $1 S, 2 S$ isomer.

Chiral HPLC (Chiralcel-OJ, Daicel, $6 \%$ iPrOH in hexanes, $0.6 \mathrm{~mL} / \mathrm{min}$ ) analysis of diol product:

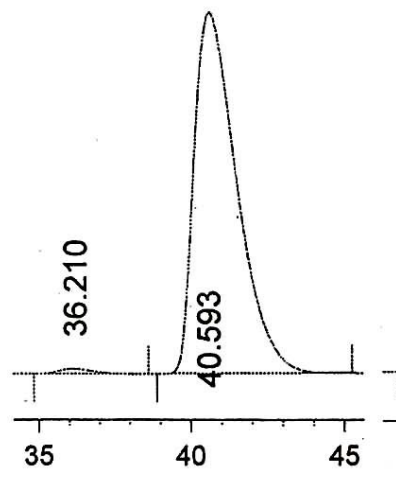

Diboration

Product

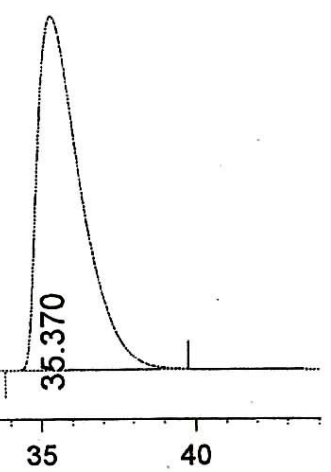

Authentic

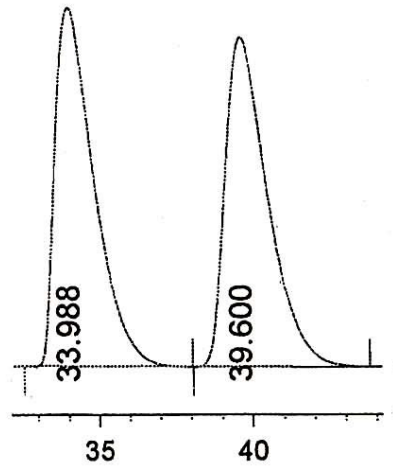

Racemic

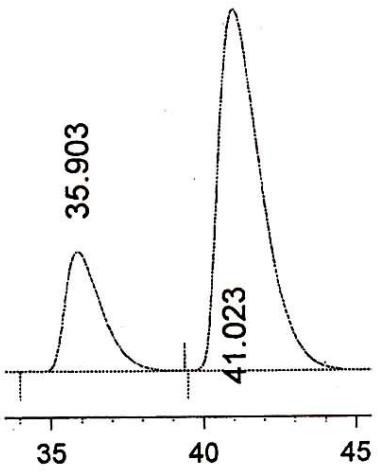

Racemic and

Diboration

Product 


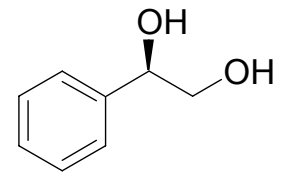

(1R)-phenyl-ethane-1, 2-diol. IR (neat): 3369 (br, s), 2925 (m), 1453 (s), $1071(\mathrm{~s}), 1027(\mathrm{~s}) \mathrm{cm}^{-1} ;{ }^{1} \mathrm{H}$ NMR: $\delta$ 7.41-7.26 (5H, m, Ph), 4.81 $(1 \mathrm{H}, \mathrm{dd}, \mathrm{J}=8.0 \mathrm{~Hz}, 3.6 \mathrm{~Hz}, \mathrm{PhCHOH}), 3.75(1 \mathrm{H}, \mathrm{dd}, \mathrm{J}=10.8 \mathrm{~Hz}, 3.6$ $\left.\mathrm{Hz}, \mathrm{CH}_{\mathrm{a}} \mathrm{H}_{\mathrm{b}} \mathrm{OH}\right), 3.65\left(1 \mathrm{H}, \mathrm{dd}, \mathrm{J}=10.8 \mathrm{~Hz}, 8.0 \mathrm{~Hz}, \mathrm{CH}_{\mathrm{a}} \mathbf{H}_{\mathrm{b}} \mathrm{OH}\right), 2.25$ (2H, br s, OH); ${ }^{13} \mathrm{C}$ NMR: $\delta 140.5,128.6,128.0,126.1,74.7,68.1$. HRMS (FAB) Calc'd for $\mathrm{C}_{8} \mathrm{H}_{10} \mathrm{O}_{2}\left(\mathrm{M}+\mathrm{NH}_{4}\right)^{+}: 156.1025$ Found $\left(\mathrm{M}+\mathrm{NH}_{4}\right)^{+}: 156.1026$.

Proof of Stereochemistry. Stereochemical ratios were determined in comparison to authentic racemic materials prepared by osmium tetraoxide dihydroxylation. Relative stereochemistry determined in comparison to ${ }^{1} \mathrm{H}$ NMR reported for the syn diol (Choudary, B. M.; Chowdari, N. S.; Jyothi, K.; Kantam, M. L. J. Am. Chem. Soc. 2002, $124,5341)$. Absolute stereochemistry established in comparison to authentic $1 R$ isomer prepared via a Sharpless asymmetric dihydroxylation (Sharpless, K. B.; Amberg, W.; Bennani, Y. L.; Crispino, G. A.; Hartung, J.; Jeong, K.-S.; Kwong, H.-L.; Morikawa, K.; Wang, Z.-M.; Xu, D.; Zhang, X.-L. J. Org. 1992, 57, 2768).

Chiral GLC ( $\beta$-dex, Supelco, $\left.130^{\circ} \mathrm{C}\right)$ analysis of diol product:

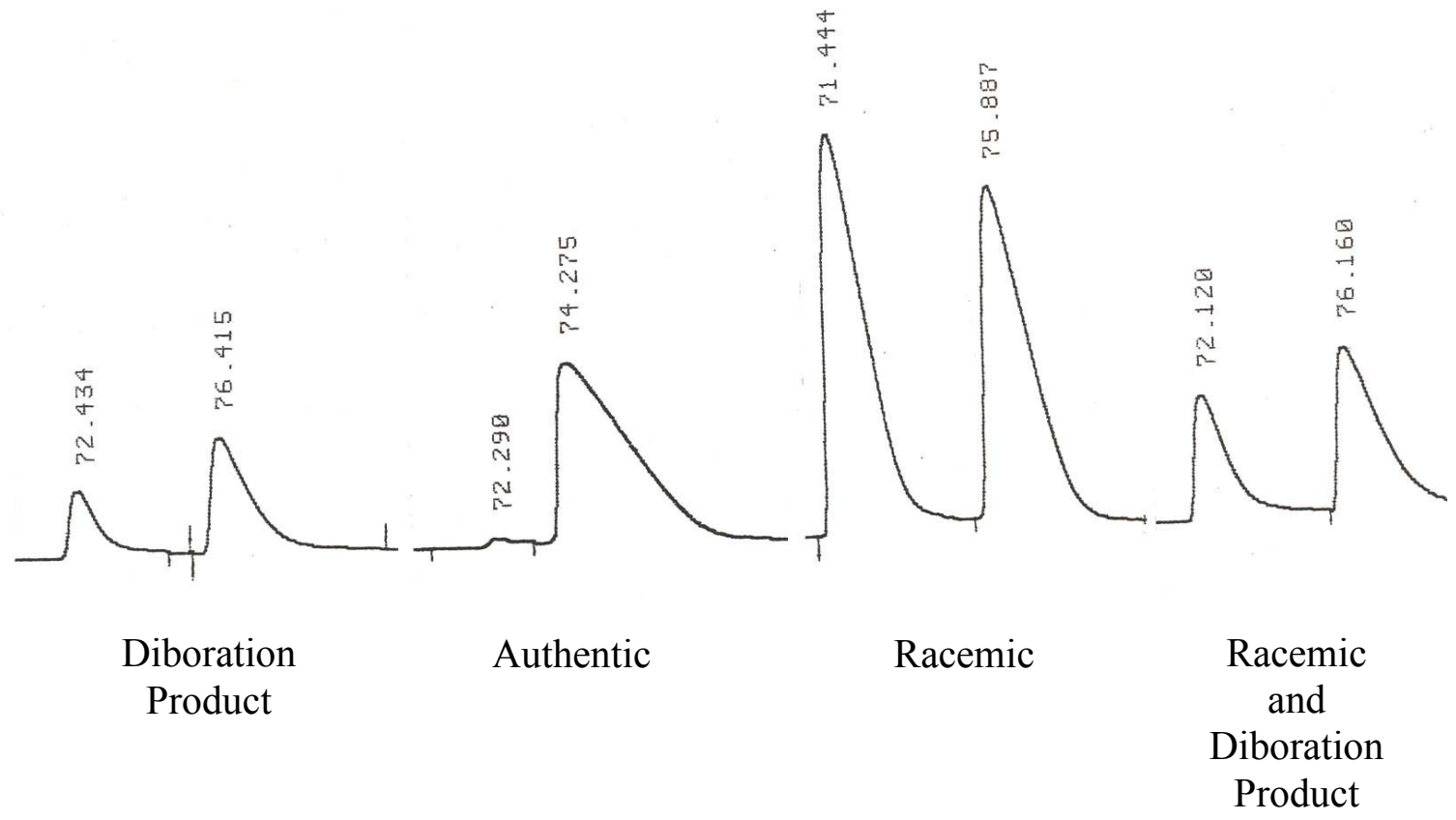


(2R)-2-phenyl-propane-1, 2-diol. IR (neat): 3375 (br, s), 2927 (m),

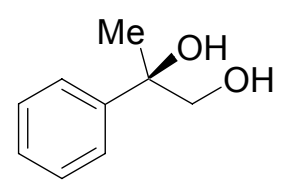
1447 (s), 1044 (s) $\mathrm{cm}^{-1}$; ${ }^{1} \mathrm{H}$ NMR: $\delta 7.43(2 \mathrm{H}, \mathrm{m}, \mathrm{Ph}), 7.36(2 \mathrm{H}, \mathrm{m}$, $\mathrm{Ph}), 7.26(1 \mathrm{H}, \mathrm{m}, \mathrm{Ph}), 3.77\left(1 \mathrm{H}, \mathrm{d}, \mathrm{J}=11.2 \mathrm{~Hz}, \mathrm{CH}_{\mathrm{a}} \mathrm{H}_{\mathrm{b}} \mathrm{OH}\right), 3.61$ $\left(1 \mathrm{H}, \mathrm{d}, \mathrm{J}=11.2 \mathrm{~Hz}, \mathrm{CH}_{\mathrm{a}} \mathrm{H}_{\mathrm{b}} \mathrm{OH}\right), 2.60(1 \mathrm{H}, \mathrm{br} \mathrm{s}, \mathrm{OH}), 1.86(1 \mathrm{H}, \mathrm{br} \mathrm{s}$, $\mathrm{OH}), 1.51\left(3 \mathrm{H}, \mathrm{s}, \mathrm{CH}_{3}\right) ;{ }^{13} \mathrm{C}$ NMR: $\delta 145.0,128.4,127.2,125.1$, 74.8, 71.1, 26.1. HRMS (FAB) Calc'd for $\mathrm{C}_{9} \mathrm{H}_{12} \mathrm{O}_{2}\left(\mathrm{M}+\mathrm{NH}_{4}\right)^{+}: 170.1181$ Found $(\mathrm{M}+$ $\left.\mathrm{NH}_{4}\right)^{+}: 170.1182$.

Proof of Stereochemistry. Stereochemical ratios were determined in comparison to authentic racemic materials prepared by osmium tetraoxide dihydroxylation. Relative stereochemistry determined in comparison to ${ }^{1} \mathrm{H}$ NMR reported for the syn diol (Choudary, B. M.; Chowdari, N. S.; Jyothi, K.; Kantam, M. L. J. Am. Chem. Soc. 2002, 124, 5341). Absolute stereochemistry established in comparison to authentic $1 R$ isomer prepared via a Sharpless asymmetric dihydroxylation (Sharpless, K. B.; Amberg, W.; Bennani, Y. L.; Crispino, G. A.; Hartung, J.; Jeong, K.-S.; Kwong, H.-L.; Morikawa, K.; Wang, Z.-M.; Xu, D.; Zhang, X.-L. J. Org. 1992, 57, 2768).

Chiral GLC ( $\beta$-dex, Supelco, $\left.120^{\circ} \mathrm{C}\right)$ analysis of diol product:

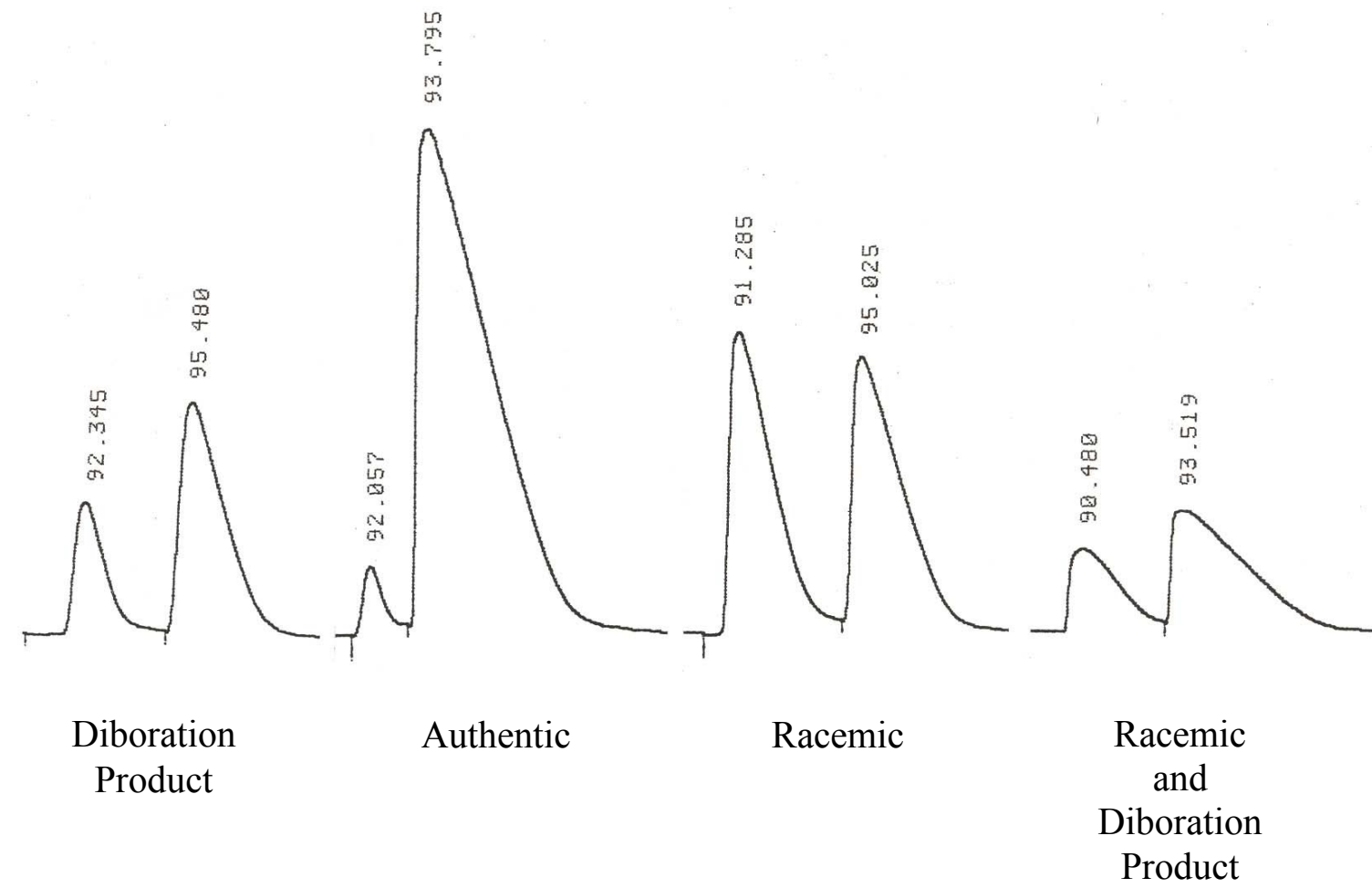




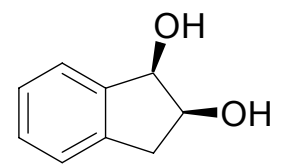

(1R, 2S)-indan-1, 2-diol. IR (neat): 3354 (br, s), 2952 (m), 2929 (s) $\mathrm{cm}^{-1}$; ${ }^{1} \mathrm{H}$ NMR: $\delta 7.4(1 \mathrm{H}, \mathrm{m}), 7.2-7.3(3 \mathrm{H}, \mathrm{m}), 5.00(1 \mathrm{H}, \mathrm{dd}, \mathrm{J}=5.6$ $\mathrm{Hz}, 5.6 \mathrm{~Hz}, \mathrm{CCHOH}), 4.51(1 \mathrm{H}, \mathrm{br} \mathrm{m}, \mathrm{CHOHCH})_{2}, 3.12(1 \mathrm{H}, \mathrm{dd}, \mathrm{J}=$ $\left.16.4 \mathrm{~Hz}, 5.6 \mathrm{~Hz}, \mathrm{CH}_{\mathbf{a}} \mathrm{H}_{\mathrm{b}}\right), 2.95\left(1 \mathrm{H}, \mathrm{dd}, \mathrm{J}=16.4 \mathrm{~Hz}, 4.4 \mathrm{~Hz}, \mathrm{CH}_{\mathrm{a}} \mathbf{H}_{\mathbf{b}}\right)$, $2.42(1 \mathrm{H}, \mathrm{d}, \mathrm{J}=5.6 \mathrm{~Hz}, \mathrm{OH}), 2.34(1 \mathrm{H}, \mathrm{d}, \mathrm{J}=4.4 \mathrm{~Hz}, \mathrm{OH}) ;{ }^{13} \mathrm{C}$ NMR: $\delta 141.9,140.1$, $128.8,127.2,125.4,125.0,75.9,73.4,38.6$. HRMS (FAB) Calc'd for $\mathrm{C}_{9} \mathrm{H}_{10} \mathrm{O}_{2}(\mathrm{M}+$ $\left.\mathrm{NH}_{4}\right)^{+}: 168.1025$ Found $\left(\mathrm{M}+\mathrm{NH}_{4}\right)^{+}: 168.1026$.

Proof of Stereochemistry. Stereochemical ratios were determined in comparison to authentic racemic materials prepared by osmium tetraoxide dihydroxylation. Relative stereochemistry determined in comparison to ${ }^{1} \mathrm{H}$ NMR reported for the syn diol (Takeshita, H.; Hatsui, T. J. Org. Chem. 1978, 43, 3080). Absolute stereochemistry established in comparison to authentic 1R, $2 \mathrm{~S}$ isomer prepared via a Sharpless asymmetric dihydroxylation (Becker, H.; King, S. B.; Taniguchi, M.; Vanhessche, K. P. M.; Sharpless, K. B. J. Org. Chem. 1995, 60, 3940).

Chiral HPLC (Chiralcel-OJ, Daicel, 7.5\% iPrOH in hexanes, $1 \mathrm{~mL} / \mathrm{min}$ )) analysis of diol product:
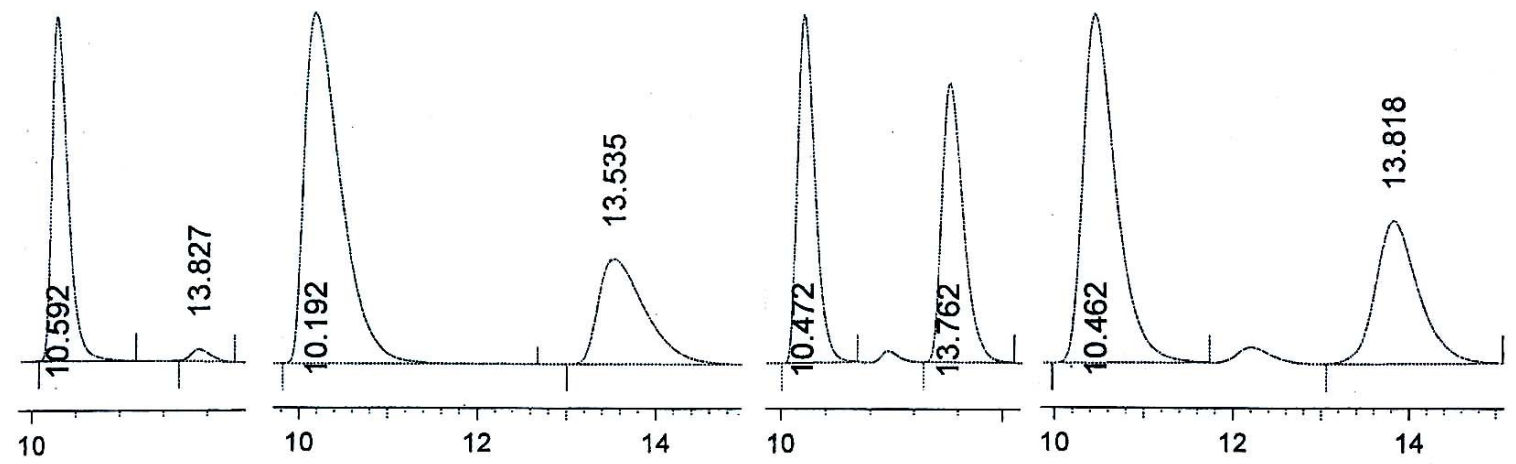

Diboration

Authentic

Racemic

Racemic

Product

and

Diboration

Product 


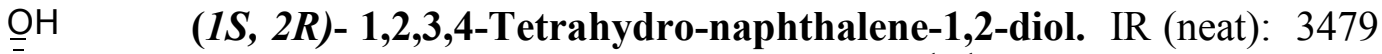
(br, s), 3054 (s), 2254 (m), 1646 (br, m) cm ${ }^{-1} ;{ }^{1} \mathrm{H}$ NMR: $\delta 7.4(1 \mathrm{H}, \mathrm{m})$, 7.2-7.3 (2H, m), $7.1(1 \mathrm{H}, \mathrm{m}), 4.68(1 \mathrm{H}, \mathrm{d}, \mathrm{J}=3.2 \mathrm{~Hz}, \mathrm{CCHOH}), 4.00$ $\left(1 \mathrm{H}, \mathrm{ddd}, \mathrm{J}=10.0 \mathrm{~Hz}, 3.6 \mathrm{~Hz}, 3.2 \mathrm{~Hz}, \mathrm{CHOHCH}_{2}\right), 2.95(1 \mathrm{H}, \mathrm{dt}, \mathrm{J}=$ 17.2 Hz, 5.6 Hz, CCH $\left.\mathbf{H}_{\mathrm{b}}\right), 2.77\left(1 \mathrm{H}, \mathrm{ddd}, \mathrm{J}=17.2 \mathrm{~Hz}, 9.2 \mathrm{~Hz}, 6.0 \mathrm{~Hz}, \mathrm{CCH}_{\mathrm{a}} \mathbf{H}_{\mathbf{b}}\right), 2.34$ $(2 \mathrm{H}$, br s, OH), 1.97-2.08 (1H, m, COHCH $), 1.86-1.95\left(1 \mathrm{H}, \mathrm{m}, \mathrm{COHCH} \mathbf{H}_{2}\right) ;{ }^{13} \mathrm{C} \mathrm{NMR:} \delta$ 136.3, 136.2, 129.9, 128.6, 128.2, 126.4, 69.9, 69.5, 26.9, 26.2. HRMS (FAB) Calc'd for $\mathrm{C}_{10} \mathrm{H}_{12} \mathrm{O}_{2}\left(\mathrm{M}+\mathrm{NH}_{4}\right)^{+}: 182.1181$ Found $\left(\mathrm{M}+\mathrm{NH}_{4}\right)^{+}: 182.1175$.

Proof of Stereochemistry. Stereochemical ratios were determined in comparison to authentic racemic materials prepared by osmium tetraoxide dihydroxylation. Relative stereochemistry determined in comparison to ${ }^{1} \mathrm{H}$ NMR reported for the syn diol (Orsini, F.; Sello, G.; Travaini, E.; Di Gennaro, P. Tetrahedron Asymmetry 2002, 13, 253). Absolute stereochemistry established in comparison to authentic 1R, $2 \mathrm{~S}$ isomer prepared via a Sharpless asymmetric dihydroxylation (Becker, H.; King, S. B.; Taniguchi, M.; Vanhessche, K. P. M.; Sharpless, K. B. J. Org. Chem. 1995, 60, 3940).

Chiral HPLC (Chiralcel-OJ, Daicel, 7.5\% iPrOH in hexanes, $0.5 \mathrm{~mL} / \mathrm{min}$ ) analysis of diol product:

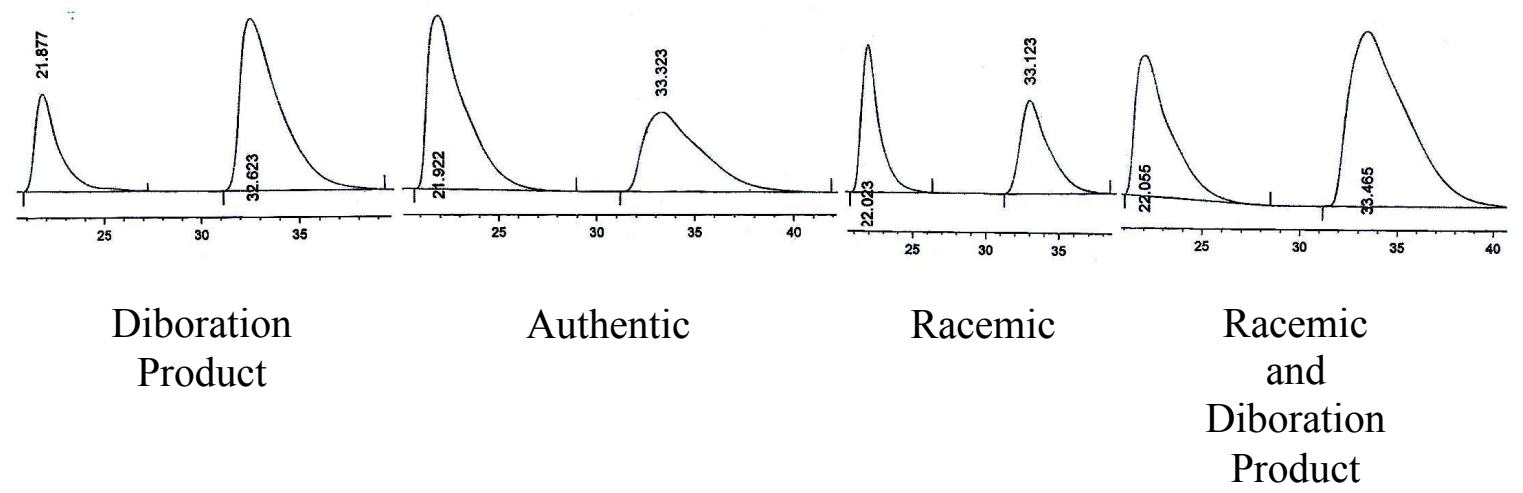




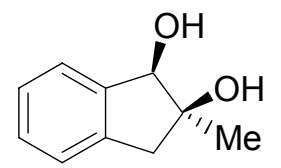

(1R, 2S)-2-Methyl-indan-1,2-diol. IR (neat): 3386 (br, s), 2960 (m), $2927(\mathrm{~s}), 2856(\mathrm{~m}) \mathrm{cm}^{-1}$; ${ }^{1} \mathrm{H}$ NMR: $\delta 7.4(1 \mathrm{H}, \mathrm{m}), 7.15-7.3(3 \mathrm{H}, \mathrm{m})$, $4.70(1 \mathrm{H}, \mathrm{d}, \mathrm{J}=6.4 \mathrm{~Hz}, \mathrm{CCHOH}), 3.04\left(1 \mathrm{H}, \mathrm{d}, \mathrm{J}=16.0 \mathrm{~Hz}, \mathrm{CH}_{\mathbf{a}} \mathrm{H}_{\mathrm{b}}\right)$, $2.94\left(1 \mathrm{H}, \mathrm{d}, \mathrm{J}=16.0 \mathrm{~Hz}, \mathrm{CH}_{\mathrm{a}} \mathbf{H}_{\mathbf{b}}\right), 2.45(1 \mathrm{H}, \mathrm{d}, \mathrm{J}=6.4 \mathrm{~Hz}, \mathrm{CHOH}), 2.25$ $\left(3 \mathrm{H}, \mathrm{s}, \mathrm{CCH}_{3} \mathrm{OH}\right), 1.47\left(1 \mathrm{H}, \mathrm{s}, \mathrm{CCH}_{3}\right) ;{ }^{13} \mathrm{C}$ NMR: $\delta 142.8,139.9,128.6,127.1,125.3$, 125.0, 80.6, 79.8, 44.7, 24.7. HRMS (FAB) Calc'd for $\mathrm{C}_{10} \mathrm{H}_{12} \mathrm{O}_{2}\left(\mathrm{M}+\mathrm{NH}_{4}\right)^{+}: 182.1181$ Found $\left(\mathrm{M}+\mathrm{NH}_{4}\right)^{+}: 182.1175$.

Proof of Stereochemistry. Stereochemical ratios were determined in comparison to authentic racemic materials prepared by osmium tetraoxide dihydroxylation. Relative stereochemistry determined in analogy to all other syn diols produced by dihydroxylation method. Absolute stereochemistry established in comparison to authentic $1 \mathrm{R}, 2 \mathrm{~S}$ isomer prepared via a Sharpless asymmetric dihydroxylation (report of analogous compound: Barboni, L.; Lambertucci, C.; Ballini, R.; Appendino, G.; Bombardelli, E. Tetrahedron Lett. 1998, 39, 7177).

Chiral HPLC (Chiralcel-OD, Daicel, 10\% iPrOH in hexanes, $1 \mathrm{~mL} / \mathrm{min}$ ) analysis of diol product:

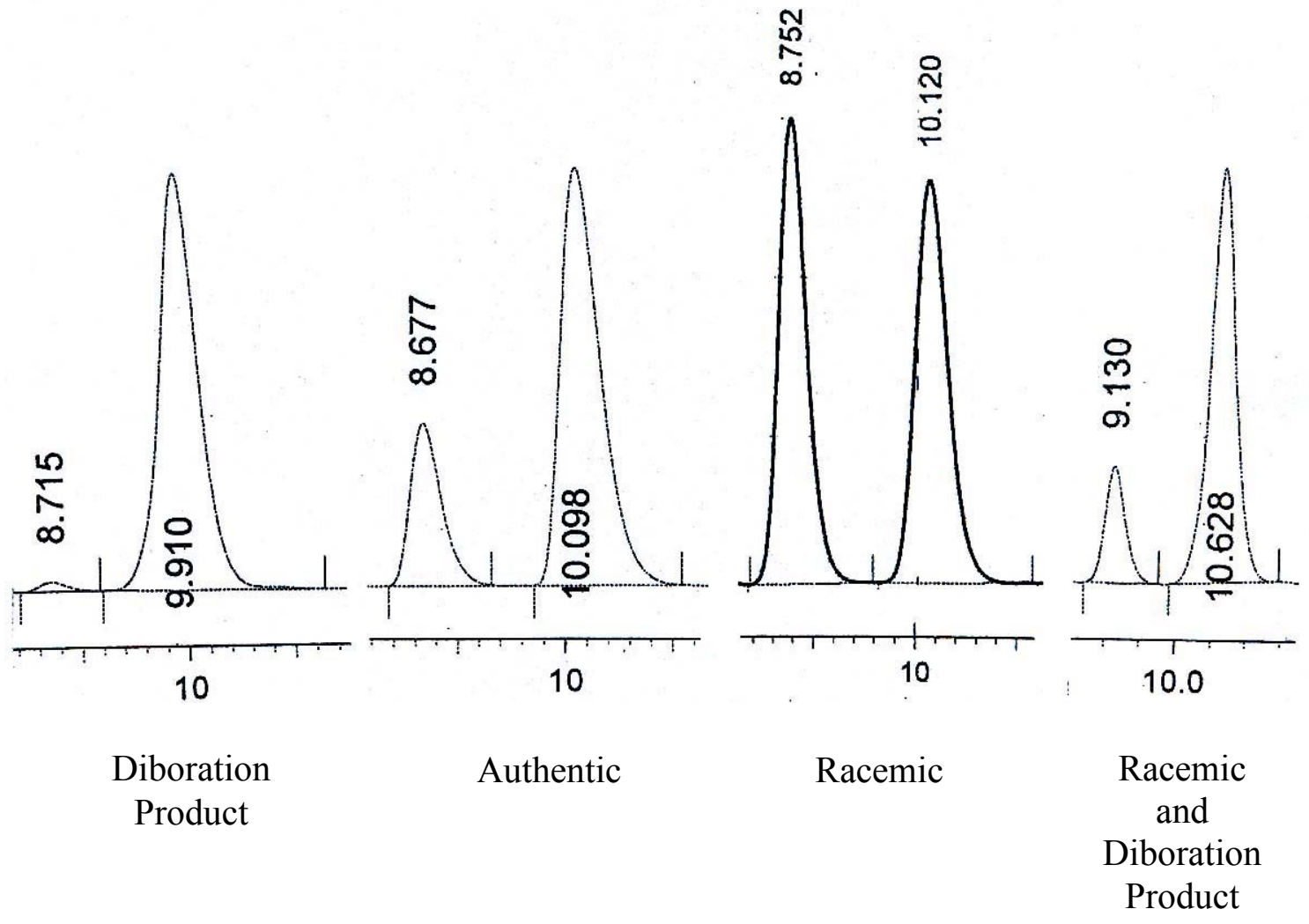




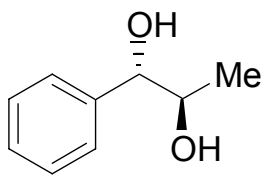

(1S, 2R)-1-phenyl-propane-1,2-diol. IR (neat): 3384 (br, s), 1455 (s), $1038(\mathrm{~s}) \mathrm{cm}^{-1} ;{ }^{1} \mathrm{H}$ NMR: $\delta$ 7.25-7.38 $(5 \mathrm{H}, \mathrm{m}$, aromatic), $4.66(1 \mathrm{H}$, $\mathrm{d}, \mathrm{J}=4.0 \mathrm{~Hz}, \operatorname{ArCHOH}), 4.00(1 \mathrm{H}, \mathrm{qd}, \mathrm{J}=6.4 \mathrm{~Hz}, 4.0 \mathrm{~Hz}$ $\left.\mathrm{CH}_{3} \mathrm{CHOH}\right), 2.40(1 \mathrm{H}$, br s, OH), $1.90(1 \mathrm{H}$, br s, OH $), 1.07(3 \mathrm{H}, \mathrm{d}, \mathrm{J}=$ $\left.6.4 \mathrm{~Hz}, \mathrm{CH}_{3}\right) ;{ }^{13} \mathrm{C}$ NMR: $\delta$ 140.7, 128.3, 127.8, 126.6, 77.5, 71.2, 17.3. HRMS (FAB) Calc'd for $\mathrm{C}_{9} \mathrm{H}_{12} \mathrm{O}_{2}\left(\mathrm{M}+\mathrm{NH}_{4}\right)^{+}: 170.1181$ Found $\left(\mathrm{M}+\mathrm{NH}_{4}\right)^{+}: 170.1181$.

Proof of Stereochemistry. Stereochemical ratios were determined in comparison to authentic racemic materials prepared by osmium tetraoxide dihydroxylation. Relative stereochemistry determined in comparison to ${ }^{1} \mathrm{H}$ NMR reported for the syn diol (Fronza, G.; Fuganti, C.; Grasselli, P.; Mele, A. J. Org. Chem. 1991, 56, 6019). Absolute stereochemistry established in comparison to authentic $1 \mathrm{R}, 2 \mathrm{~S}$ isomer prepared via a Sharpless asymmetric dihydroxylation (Becker, H.; King, S. B.; Taniguchi, M.; Vanhessche, K. P. M.; Sharpless, K. B. J. Org. Chem. 1995, 60, 3940).

Chiral GLC ( $\beta$-dex, Supelco, $\left.140{ }^{\circ} \mathrm{C}\right)$ analysis of diol product:

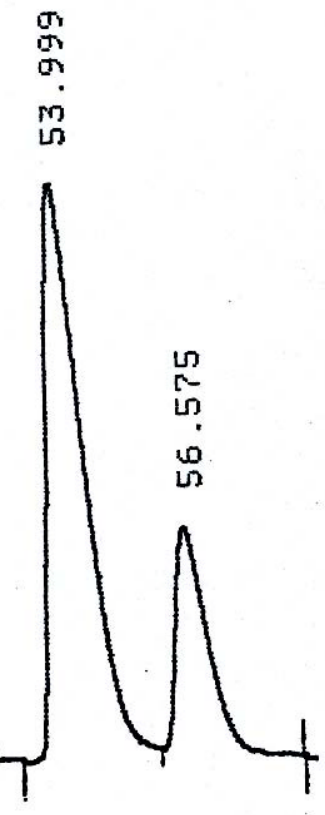

Diboration

Product

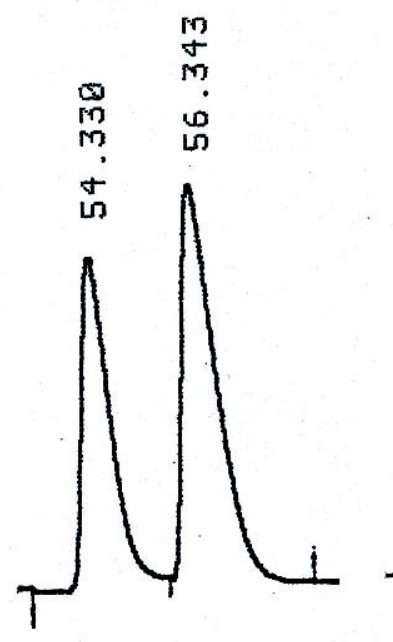

Authentic

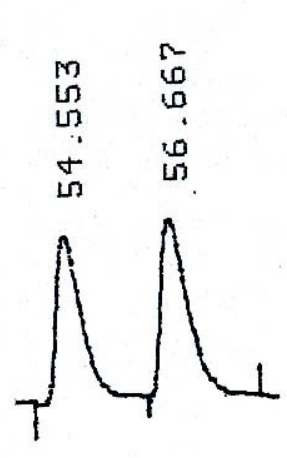

Racemic

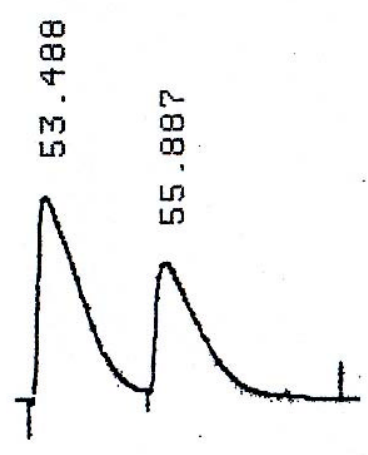

Racemic and

Diboration

Product 
<smiles>COc1ccc(C(O)C(C)O)cc1</smiles>

(1R, 2R)-1-(4-Methoxy-phenyl)-propane-1,2-diol. IR (neat): 3452 (br, s), 1638 (br, m), $1515(\mathrm{~m}) \mathrm{cm}^{-1} ;{ }^{1} \mathrm{H}$ NMR: $\delta 7.23(2 \mathrm{H}$, $\mathrm{m}$, aromatic), $6.86(2 \mathrm{H}, \mathrm{m}$, aromatic $), 4.29(1 \mathrm{H}, \mathrm{d}, \mathrm{J}=7.6 \mathrm{~Hz}$, $\mathrm{ArCHOH}), 3.75-3.86\left(1 \mathrm{H}, \mathrm{m}, \mathrm{CH}_{3} \mathrm{CHOH}\right), 3.79\left(3 \mathrm{H}, \mathrm{s}, \mathrm{OCH}_{3}\right)$, $2.59(1 \mathrm{H}$, broad s, OH $), 2.54(1 \mathrm{H}, \mathrm{s}, \mathrm{OH}), 1.01\left(3 \mathrm{H}, \mathrm{d}, \mathrm{J}=6.4 \mathrm{~Hz}, \mathrm{CH}_{3}\right) ;{ }^{13} \mathrm{C}$ NMR: $\delta$ 159.4, 133.1, 128.0, 113.9, 79.1, 72.2, 55.3, 18.7. HRMS (FAB) Calc'd for $\mathrm{C}_{10} \mathrm{H}_{14} \mathrm{O}_{3}(\mathrm{M}$ $\left.+\mathrm{NH}_{4}\right)^{+}: 200.1287$ Found $\left(\mathrm{M}+\mathrm{NH}_{4}\right)^{+}: 200.1282$.

Proof of Stereochemistry. Stereochemical ratios were determined in comparison to authentic racemic materials prepared by osmium tetraoxide dihydroxylation. Relative stereochemistry determined in comparison to ${ }^{1} \mathrm{H}$ NMR reported for the syn diol (Mohan, R. S.; Whalen, D. L. J. Org. Chem. 1993, 58, 2663). Absolute stereochemistry established in comparison to authentic $1 \mathrm{R}, 2 \mathrm{R}$ isomer prepared via Sharpless asymmetric dihydroxylation (analogous to $\beta$-Methylstryene: Norrby, P-O.; Becker, H.; Sharpless, K. B. J. Am. Chem. Soc. 1996, 118, 35).

Chiral GLC ( $\beta$-dex, Supelco, $\left.160^{\circ} \mathrm{C}\right)$ analysis of diol product:

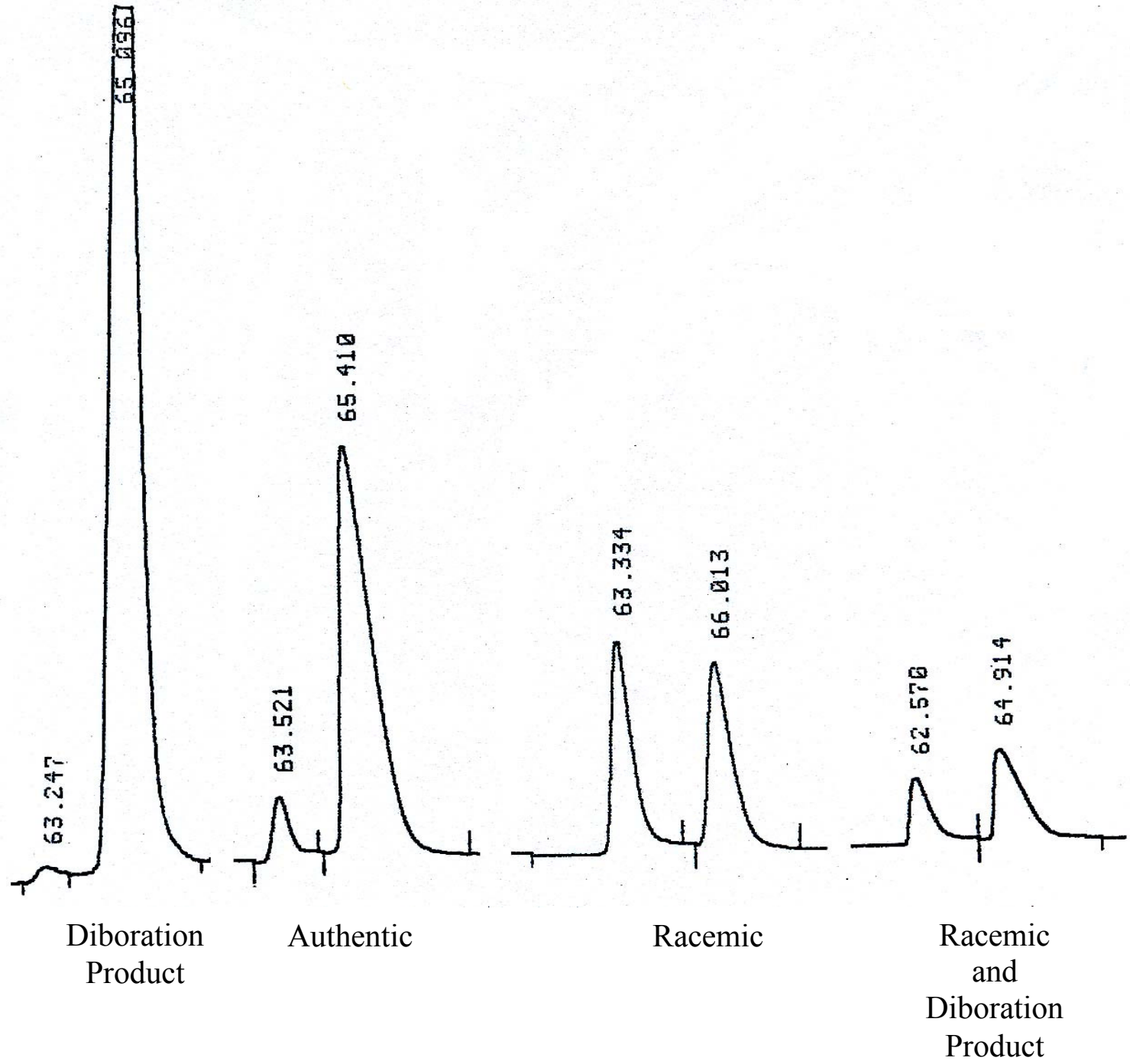




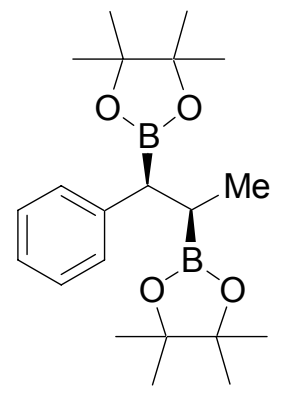

(1S, 2R)-di(pinacolatoboryl) 1-phenyl-propane. ${ }^{1} \mathrm{H}$ NMR: $\delta$ 7.0-7.25 $(5 \mathrm{H}, \mathrm{m}$, aromatic), $2.20(1 \mathrm{H}, \mathrm{d}, \mathrm{J}=12.0 \mathrm{~Hz}, \mathrm{ArCHB}), 1.45-1.57(1 \mathrm{H}$, $\left.\mathrm{m}, \mathrm{CH}_{3} \mathrm{CHB}\right), 1.24\left(12 \mathrm{H}, \mathrm{s}, \mathrm{OCCH}_{3}\right), 1.15\left(6 \mathrm{H}, \mathrm{s}, \mathrm{OCCH}_{3}\right), 1.14(6 \mathrm{H}$, $\left.\mathrm{s}, \mathrm{OCCH}_{3}\right), 0.73\left(3 \mathrm{H}, \mathrm{d}, \mathrm{J}=7.6 \mathrm{~Hz}, \mathrm{CH}_{3}\right) ;{ }^{13} \mathrm{C} \mathrm{NMR}: \delta 142.9,128.9$, $128.0,124.9,83.1,83.0,25.0,24.9,24.8,24.5,24.2,14.5$.

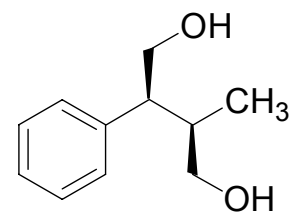

(2R, 3R)-2-Methyl-3-phenyl-butane-1,4-diol. ${ }^{1} \mathrm{H}$ NMR: $\delta$ 7.1-7.35 $(5 \mathrm{H}, \mathrm{m}), 3.89\left(1 \mathrm{H}, \mathrm{dd}, \mathrm{J}=10.8 \mathrm{~Hz}, 6.4 \mathrm{~Hz}, \operatorname{ArCHCH}_{\mathrm{a}} \mathrm{H}_{\mathrm{b}} \mathrm{OH}\right), 3.85$ $\left(1 \mathrm{H}, \mathrm{dd}, \mathrm{J}=10.8 \mathrm{~Hz}, 5.6 \mathrm{~Hz}, \operatorname{ArCHCH}_{\mathrm{a}} \mathbf{H}_{\mathbf{b}} \mathrm{OH}\right), 3.70(1 \mathrm{H}, \mathrm{dd}, \mathrm{J}=11.2$ $\left.\mathrm{Hz}, 4.4 \mathrm{~Hz}, \mathrm{CH}_{3} \mathrm{CHCH}_{\mathrm{a}} \mathrm{CH}_{\mathrm{b}} \mathrm{OH}\right), 3.58(1 \mathrm{H}, \mathrm{dd}, \mathrm{J}=11.2 \mathrm{~Hz}, 6.4 \mathrm{~Hz}$, $\left.\mathrm{CH}_{3} \mathrm{CHCH}_{\mathrm{a}} \mathrm{CH}_{\mathrm{b}} \mathrm{OH}\right), 2.71(1 \mathrm{H}, \mathrm{dt}, \mathrm{J}=8.8 \mathrm{~Hz}, 6.4 \mathrm{~Hz}, \operatorname{ArCH}), 2.62$ (2H, br s, OH), 1.97-2.10 (1H, br m, $\left.\mathbf{C H C H}_{3}\right), 0.74\left(3 \mathrm{H}, \mathrm{d}, \mathrm{J}=6.8 \mathrm{~Hz}, \mathrm{CH}_{3}\right) ;{ }^{13} \mathrm{C}$ NMR: $\delta 141.8,128.5,128.3,126.6,66.7,65.8,52.0,38.6,15.8$. HRMS (FAB) Calc'd for $\mathrm{C}_{11} \mathrm{H}_{16} \mathrm{O}_{2}\left(\mathrm{M}+\mathrm{NH}_{4}\right)^{+}: 198.1494$ Found $\left(\mathrm{M}+\mathrm{NH}_{4}\right)^{+}: 198.1493$. 